\title{
Cell membranes composition is defined in ER and their restitution proceed by en bloc fusion of ER generated transport vesicles
}

\author{
Amalia Slomiany*, Bronislaw L. Slomiany \\ Research Center, University of Medicine and Dentistry of New Jersey, Newark, USA; *Corresponding Author: slomiabr@umdnj.edu
}

Received 28 September 2010; revised 19 October 2010; accepted 25 October 2010

\begin{abstract}
The synthesis of endoplasmic reticulum (ER)derived transport vesicles is dictated by the contents and derivation of the cellular cytosol. The ER transport vesicles synthesized in the presence of gastric epithelial cells cytosol are destined for en bloc fusion with apical epithelial membrane, whereas those generated in hepatocytes-derived cytosol are destined for en bloc fusion with basolateral membrane. Moreover, during assembly of the dominant fraction of the apical or basolateral transport vesicles, a substantial fraction of the vesicles is produced that fuses with endosomes, and the vesicles with still unknown destination that remain in cytosol. The process of ER vesicles synthesis is blocked by RNase treatment, whereas Golgi vesicles assembly is not affected. The experiments indicate that transport vesicles' membrane composition and fidelity of its construction is defined in ER. The process involves synchronous membrane lipid synthesis, cotranslational intercalation of integral membrane proteins and containment of the vesicular cargo.
\end{abstract}

Keywords: Membrane Biogenesis; Repair Fidelity; Vesicular Transport; En Bloc Fusion

\section{INTRODUCTION}

Numerous comprehensive reviews of cellular transport provide detailed view of the proteins involved in vesicles targeting and membrane fusion [1-8]. Nevertheless, the fundamental problem of membrane fusion still remains a puzzle that has yet to be solved in any biological system $[9,10]$. Thus far, the envisioned schemes do not provide complete picture and explain how the diversity of cellular membranous structures is maintained or how the cell membranes are repaired and their compo- nents restored $[3,10,11]$. In the proposed concepts of the vesicular transport, the endoplasmic reticulum (ER) is depicted as a protein synthetic and folding site apart from its role in the vesicular membrane lipid synthesis and membrane assembly that includes insertion of the integral proteins dedicated for the specific vesicle and destination site. In our view, the independent of each other lipid assembly into the membrane and protein translation and the capture of preassembled membranes' proteins $[2-5,12,13]$ is lacking precision that is critical to retain cell characteristic features $[2-4,14]$. It is also not plausible that preassembled or recycled vesicular carriers could attain the degree of the reproducible fidelity that is achieved when protein translation, membrane lipid synthesis and vesicle formation occur in the synchrony and is confined to a specific segment of ER. Moreover, the lipid microenvironment that is required for the precise function of membrane integral protein can only be reproduced when the process involves simultaneous membrane lipids synthesis, protein intercalation and en bloc membrane replacement [14-17].

Our previously published studies of apical transport in gastric mucosal epithelial cells demonstrated synchrony in the assembly of transport vesicles membrane and synthesis of apoprotein (apomucin) cargo [18,19]. Gastric mucosal epithelium elaborates enormous quantity of glycoprotein (mucin) and requires just as large synthetic capacity to generate membranes for the vesicles to move the cargo from ER to Golgi and to the apical cell membrane. In the conducted studies by tracing labeled proteins and lipids constituting the vesicular membrane and secretory cargo we revealed that in ER newly synthesized apomucin was packaged into vesicles generated from newly synthesized membrane that contained apical membrane-specific integral membrane proteins and lipids [15-21]. The newly ER-synthesized transporting units fused with Golgi and underwent further processing consisting of cargo glycosylation and vesicles' membrane destination-specific phosphatidylinositol- (PI) and 
the ceramides (Cer) modification. PI was phosphorylated to phosphatidylinositol 3- and 4-phosphates (PI3P and PI4P) and Cer used for assembly of sphingomyelin (SM) and glycosphingolipids (GSL) [15-17]. The vesicles containing GPI-anchored mucin binding protein (MBP), PI3P and mucin cargo fused with apical cell membrane and the Golgi vesicle's membrane incorporated en bloc into apical epithelial continuum [15-17]. The en bloc incorporation of the vesicular membrane into apical membrane continuum was manifested by inclusion of the labeled lipids that comprised the vesicle into the apical membrane and was coupled with formation of lyso-PI3P and generation of arachidonate [15]. Moreover, the Golgi-modified vesicles that would not fuse with apical membrane contained PI4P and portion displayed affinity for endosomes [16,17].

Our studies presented here demonstrate the transfer of ER-initiated, radiolabeled vesicular membranes to basolateral site of the hepatocytes. The hepatocytes ER-derived transport vesicles fuse with basolateral membrane contain PIPs but their membranes are devoid of glycosphingolipids and PI3P, and they do not fuse with gastric epithelial apical membrane. Compositional differences between lipids of ER membrane, apical and basolateral cell membranes [18-20] and en bloc intercalation of lipids and membrane protein into specific site of the cell membrane which is synchronized with apical or basolateral secretion [15-17] support the concept that transport vesicles are precisely fashioned in ER and delivered to the specific site for the precise membrane restitution and the cell function. The stereotypic view that cellular vesicles capture protein, discharge it and return for the next tour of transport [2] is insensitive to the functional relevance of the precise intercalation of membrane proteins within specific membrane lipids. The precise intercalation of membrane-spanning proteins can only be achieved during synchronized protein translation and ER-membrane lipids biogenesis.

\section{MATERIALS AND METHODS}

\subsection{Materials}

Radiolabeled precursors of phospholipids, glycolipids, glycoproteins and other radiochemicals were purchased from New England Nuclear (Boston, MA). Phospholipid standards were from Avanti (Birmingham, AL) Matreya (Pleasant Gap, PA) or prepared in our laboratory. Creatine phosphokinase, phenylmethylsulfonyl fluoride (PMSF), aprotinin, pepstatin, leupeptin, ATP, CTP, GTP, fatty acyl CoA, glycerol 3-phosphate, RNase, and RNase free sucrose were purchased from Sigma Chemicals (St. Louis, MO). Polyacrylamide gel electrophoresis reagents were from Bio-Rad (Rockville Centre, NY). All other chemicals and reagents were purchased from J.T. Baker Chemical Co. (Phillipsburg, PA) Fisher Scientific (Springfield, NJ) and VWR Scientific (Piscataway, NJ). The anti-rat albumin antibodies were purchased, and the antimucin antibodies were prepared in our laboratory [22].

\subsection{Liver Perfusion Buffers}

a) Buffered saline, with $10 \mathrm{mM}$ potassium phosphate, $\mathrm{pH} 6.8, \mathrm{~b})$ buffered saline containing $0.5 \mathrm{mM} \mathrm{MgCl}_{2}$ and $\left.0.5 \mathrm{mM} \mathrm{MgSO}_{4}, \mathrm{c}\right)$ buffered saline $(100 \mathrm{ml})$ containing $66 \mathrm{mg}$ collagenase, $80 \mathrm{mg}$ hyaluronidase, and $2 \mathrm{~g}$ of albumin, d) MSB, pH 6.9 buffer consisting of 0.1 Pipes, pH 6.9, 2.0 M glycerol, $1 \mathrm{mM} \mathrm{Mg}$ acetate, $0.5 \mathrm{mM}$ EGTA and mixture of protease inhibitors consisting of leupeptin, aprotinin and PMSF, e) MSB buffer containing $0.2 \%$ Triton $\mathrm{X} 100, \mathrm{f}) 50 \mathrm{mM}$ TRIS-HCl, pH 7.4 con-

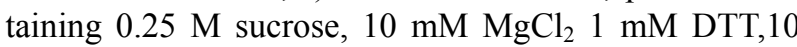
$\mathrm{mg} / \mathrm{ml}$ leupeptin and $2 \mathrm{mM}$ PMSF.

\subsection{Perfusion of Rat Liver and Isolation of Hepatocytes}

The abdomen of anestetized rat was open and liver cannulated and perfused with $50 \mathrm{ml}$ of ice cold Hanks Balanced Salts (Sigma) without $\mathrm{Ca}^{2+}$ containing $20 \mathrm{mg}$ collagenase, type IV, $20 \mathrm{mg}$ hyaluronidase, $1 \mathrm{~g}$ defatted albumin, and $3 \mathrm{~g}$ of heparin. After initial perfusion, the liver was removed from animal's abdomen, cut into slices and incubated with cold Hanks solution. Thus prepared slices were subjected to incubation in tissue incubator in $95 \% \mathrm{O}_{2}$ and $5 \% \mathrm{CO}_{2}$ for $40 \mathrm{~min}$, at $37^{\circ} \mathrm{C}$. The slices were broken up with rubber policemen, incubated for additional $10 \mathrm{~min}$ and filtered through nylon mesh that separated single cells from larger debris. The cells were then centrifuged at 50xg for $2 \mathrm{~min}$, washed twice with the enzyme-free Hanks medium, twice with the Minimum Essential Medium and counted in hemocytometer. Thus prepared cells were used for preparation of nuclei [24] subcellular organelles, cell cytosol $[15,19,20]$ and cellular membranes $[15,16]$. In the experiments dedicated to the determination of lipid synthesis with cell cytosol derived from gastric epithelial cells, or RNase treated cytosol, the preparations of ER, Golgi, endosomes or nuclei were additionally rinsed with PBS and urea-PBS in order to remove the residual cytosolic proteins. Thus prepared subcellular organelles and membranes were used for experiments on transport vesicles synthesis and fusion [15] and preparation of outer nuclear membrane (ONM) and the Inner Nuclear Membrane (INM) [24]. The synthesis of phosphatidylinositides, phospholipids and protein was determined using radiolabeled $\left[{ }^{3} \mathrm{H}\right]$ inositol, $\left[{ }^{3} \mathrm{H}\right]$ arachidonate, $\left[{ }^{3} \mathrm{H}\right]$ choline, $\left[{ }^{3} \mathrm{H}\right]$ serine, $\left[{ }^{3} \mathrm{H}\right]$ palmitate and $\left[{ }^{32} \mathrm{P}\right] \mathrm{ATP}[15-20]$. The 
synthesis of transport vesicles (ER, Golgi) was performed in medium containing cytosol at concentration of $5 \mathrm{mg}$ protein $/ \mathrm{ml}$ of incubation mixture enriched with 50 mM ATP, $250 \mathrm{mM}$ CTP, $50 \mathrm{mM}$ GTP, $5 \mathrm{mM}$ creatine phosphate, $8.0 \mathrm{IU} / \mathrm{ml}$ creatine kinase, and where indicated $25 \mathrm{mg} / \mathrm{ml}$ RNase, $10 \mathrm{mM}$ UDP-Glc and $10 \mathrm{mM}$ palmitoyl CoA [15-20]. The same preparation of cytosol was used in the experiments with nuclear membranes [24].

\subsection{Isolation and Separation of ER, Golgi, Endosomes, Cellular and Outer and Inner Nuclear Membranes}

One volume of the isolated hepatocytes was homogenized in 8 volumes of $10 \mathrm{mM}$ potassium phosphate buffer, $\mathrm{pH} 6.8,1.3 \mathrm{M}$ sucrose and $1 \mathrm{mM} \mathrm{MgCl}_{2}$ to rupture at least $80 \%$ of cells [24]. The unbroken cells were removed by centrifugation at 50xg for $3 \mathrm{~min}$, and the homogenate centrifuged for $15 \mathrm{~min}$ at $1000 \mathrm{~g}$. The soluble cellular material was saved for isolation of cellular organelles and cytosol while the nuclear pellet was processed further and the Outer and Inner Nuclear Membranes (ONM, INM) were collected [24].

The isolated on sucrose gradient hepatocytes membranes were used for fusion experiments with Golgi-derived transport vesicles. Following fusion, the apical portions of the membrane were separated by dissolving the basolateral membranes with aid of cold $0.2 \%$ Triton X100, and recovering the apical fraction enriched in glycosphingolipids and glycoprotein through floatation on sucrose gradient $[17,23]$.

\subsection{Preparation of Transport-Active Cytosol}

The viable hepatocytes (for hepatocytes cytosol) or gastric mucosal epithelial cells (for gastric epithelial cells cytosol) were homogenized for $10 \mathrm{sec}$ at $600 \mathrm{rpm}$ in 3 volumes of buffer containing $0.25 \mathrm{M}$ sucrose; 50 $\mathrm{mM}$ TRIS-HCl (pH 7.4); 25 mM magnesium acetate and $10 \mathrm{mM}$ each of aprotinin, leupeptin, chemostatin; and 1 $\mathrm{mM}$ phenylmethylsulfonylfluoride. The homogenate was centrifuged at $5,000 \mathrm{x} \mathrm{g}$ for $15 \mathrm{~min}$, the supernatant diluted with 2 volumes of homogenization buffer, and centrifuged at $10,000 \mathrm{~g}$ for $20 \mathrm{~min}$. The resulting supernatant was then subjected to centrifugation at $100,000 \mathrm{~g}$ for $1 \mathrm{~h}$. Thus obtained soluble fraction was adjusted to 15 to 18 $\mathrm{mg}$ protein $/ \mathrm{ml}$; admixed with an ATP generating system consisting of $40 \mathrm{mM}$ ATP, $200 \mathrm{mM}$ creatine phosphate, 2,000 units/ml creatine phosphokinase, and referred to as transport active cytosol or active cytosol.

\subsection{Preparation of Cellular Membranes}

The cell membranes and subcellular organelles (ER,
Golgi) were recovered from the sediment resulting from centrifugation at $100,000 \mathrm{~g}$. After removing supernatant, the transport active cytosol, the residue was suspended in buffer containing 0.2 M PIPES ( $\mathrm{pH}$ 6.9), 2 M glycerol, $1 \mathrm{mM}$ EGTA and $1 \mathrm{mM}$ magnesium acetate and applied on the top of discontinuous gradient of 2.0/1.5/1.3/1.0 M sucrose and centrifuged at $100,000 \mathrm{~g}$ for $16 \mathrm{~h}$. The cell membranes were recovered from 1.0 M sucrose, Smooth Endoplasmic Reticulum (SER) from 1.3 M sucrose, RER from 1.5 M sucrose and Golgi from the top of the 2.0 M sucrose. Each sucrose-separated fraction was subjected to further purification. The cell membranes were washed with original Pipes buffer and centrifuged at 3,000 rpm for $2 \mathrm{~min}$. To separate apical epithelial membranes, the buffer was adjusted with $0.2 \%$ Triton $\mathrm{X}-100$ and the mixture incubated at $4^{\circ} \mathrm{C}$ for $5 \mathrm{~min}$ [23]. This treatment resulted in dissolving the membranes that were free of glycosphingolipids and membrane glycoproteins. The membranes enriched in glycosphingolipids and glycoproteins were recovered by low speed centrifugation at 3,000 rpm for $2 \mathrm{~min}$. Thus prepared fractions of cell membranes were used in fusion experiments with Golgi-derived transport vesicles. The endosomes were isolated from the mitochondria-enriched fraction that sedimented at 10,000g [17].

\subsection{Generation and Purification of ER- and Golgi-derived Transport Vesicles}

ER- and Golgi-derived transport vesicles were generated in the presence of radiolabeled precursors according to procedure described previously [15-20]. The ER or Golgi membranes incubated with cytosol, ATP-generating system, UTP, CTP GTP, fatty acyl CoA and water soluble cold or radiolabeled lipids precursors were incubated for $30 \mathrm{~min}$ at $37^{\circ} \mathrm{C}$, centrifuged over $0.3 \mathrm{M}$ sucrose and treated with stripping buffer at $2{ }^{\circ} \mathrm{C}$ for $15 \mathrm{~min}$ followed by centrifugation at $10,000 \mathrm{~g}$ for $10 \mathrm{~min}$. to separate transport vesicles from ER or Golgi membranes. The separated from maternal membranes transport vesicles were recovered from the supernatant resulting from centrifugation of the supernatant mixture at $150,000 \mathrm{~g}$ for $60 \mathrm{~min}$. The crude fraction of the transport vesicles was suspended in 55\% sucrose, overlaid with $55-30 \%$ gradient and centrifuged at $150,000 \mathrm{~g}$ for $16 \mathrm{~h}$. The purified transport vesicles were recovered from the gradients as shown in Figure 2.

\subsection{Fusion Assays of Golgi-derived Transport Vesicles with Cell Membranes}

One volume of Golgi transport vesicles (1.3-1.5 protein $/ \mathrm{ml}$ ) was suspended in one volume of active cytosol (15mg protein $/ \mathrm{ml}$ and added to one volume of cell 
membranes $(8 \mathrm{mg}$ protein $/ \mathrm{ml}$ of whole cell membranes or $2 \mathrm{mg}$ protein $/ \mathrm{ml}$ of apical epithelial membranes). The reaction was allowed to proceed from $0-30 \mathrm{~min}$ at $4{ }^{\circ} \mathrm{C}$ (control) and at $37^{\circ} \mathrm{C}$ in the presence of ATP regenerating system consisting of $40 \mathrm{mM}$ ATP, $200 \mathrm{mM}$ creatine phosphate, 2,000 units $/ \mathrm{ml}$ of creatine phosphokinase, or in the ATP depleting system containing $5 \mathrm{mM}$ glucose and 500 units $/ \mathrm{ml}$ hexokinase. After incubation, the membranes were recovered by centrifugation through three volumes of $0.5 \mathrm{M}$ sucrose at 3,000 rpm for $5 \mathrm{~min}$. The free vesicles were recovered from the supernatant and used in fusion experiments with endosomes. The cell membranes sedimented through sucrose were washed with $25 \mathrm{mM}$ Hepes-KOH buffer and treated for 5 min with $0.2 \%$ Triton X-100 at $4{ }^{\circ} \mathrm{C}$. The soluble fraction of the membrane was recovered in supernatant, whereas apical the glycosphingolipids- and glycoprotein- containing membranes were in sediment. In the experiments estimating en bloc fusion of transport vesicles with the membrane, the associated but not fused vesicles were released from the membrane by subjecting the membrane fraction to treatment with $2 \mathrm{M}$ urea for 30 $\min$ at $4^{\circ} \mathrm{C}$ and then the recovered membranes were centrifuged through $0.5 \mathrm{M}$ sucrose, washed and subjected to lipid analysis.

\subsection{Lipid Analysis}

Preparations of ER, Golgi membranes, their transport vesicles, the membranes recovered after incubation with Golgi vesicles and the endosomes incubated with the vesicles recovered after fusion with cell membranes were subjected to lipid analysis. The lipid extracts and high performance thin layer chromatography were performed exactly as described in our previous studies [15-20]. Formation of PIPs was ascertained by performing ER transport vesicles synthesis in the presence of $\left[{ }^{3} \mathrm{H}\right]$ inositol or $\left[{ }^{3} \mathrm{H}\right]$ arachidonate followed by Golgi vesicles formation either in the presence or the absence of $\left[{ }^{32} \mathrm{P}\right]$ ATP [15]. Because the fusion of transport vesicles was aided by $\mathrm{PLA}_{2}$-specific hydrolysis of the membrane lipids, the resulting membranes were analyzed to demonstrate release of the $\left[{ }^{3} \mathrm{H}\right]$ arachidonate and formation of lysophosphatidylinositols (LPIs).

\section{RESULTS}

Our studies on homeostatic restitution of cellular and subcellular membranes showed that vesicular intracellular transport is engaged in systematic and coordinated replacement of lipids and proteins in the membranes of the secretory, non-dividing epithelial cells, whereas restitution of lipids in the nuclear envelope biomembrane proceeds without formation of transport vesicles [15-17,
24].

Here, we concentrated on deciphering the assembly of the biomembrane that delivers the ER products to Golgi and then forms Golgi transport vesicles destined to basolateral membrane of the hepatocytes. The biomembrane of the hepatocytes-derived ER transport vesicles is synthesized with the same phospholipids as gastric epithelial ER vesicles, consists of phosphatidylcholine (PC), phosphatidylinositol (PI), phosphatidylethanolamine (PE) and ceramide (Cer) and is free of sphingomyelin (SM) and glycosphingolipids s (GSL) (Figure 1).

The assembly of the ER transport vesicles in hepatocytes and gastric epithelial mucosal cells was inhibited by the treatment of the cell cytosol with RNase (Figure 2). The attributes of the ER-initiated biomembrane and Golgi transport vesicles derived from it are dictated by the cytosol derivation (Figure 3). As demonstrated in Figure 3, over $40 \%$ of Golgi transport vesicles that are initiated in ER in the presence of hepatocytes-derived cytosol fuse with basolateral membrane, only $11 \%$ with apical membranes and remaining vesicles stay in the cytosol.

The experiments with ER transport vesicles generated in the presence of gastric mucosal epithelial cell cytosol demonstrate that $46 \%$ of the derived Golgi vesicles fuse with apical membranes and only trace $(1.5 \%)$ fuses with basolateral membrane. In both systems about $50 \%$ of the Golgi transport vesicles stay unbound in the cytosol. Upon further incubation of the vesicles remaining in the cytosol, prominent portion of the fraction (20\%) fuses with endosomes, whereas $24-27 \%$ of the transport vesicles still remain free. Neither complete mixture of Golgi transport vesicles prior reaction with cellular membranes and endosomes, nor the portion remaining after reaction with those membranes displays any affinity for the

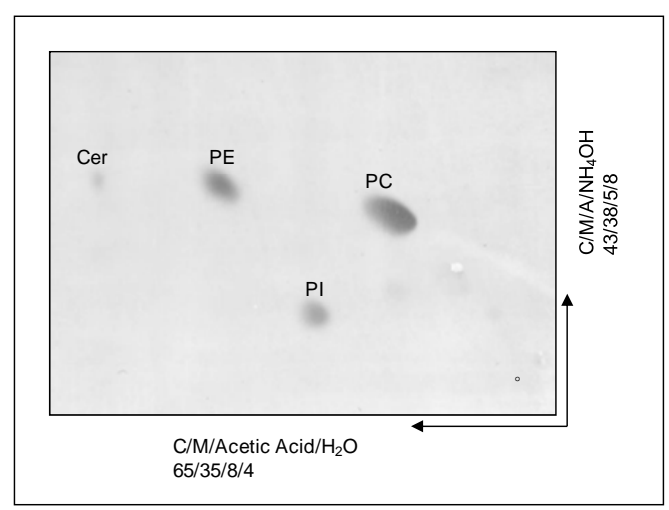

Figure 1. High Performance Thin Layer chromatography (HPTLC) of the lipids extracted from the hepatocytes ER-derived transport vesicles. The transport vesicle membrane consisted of phosphatidylcholine (PC), phosphatidylinositol (PI), phosphatidylethanolamine (PE) and ceramide (Cer). Neither sphingomyelin (SM) nor glycosphingolipids (GSL) were 
discernable.

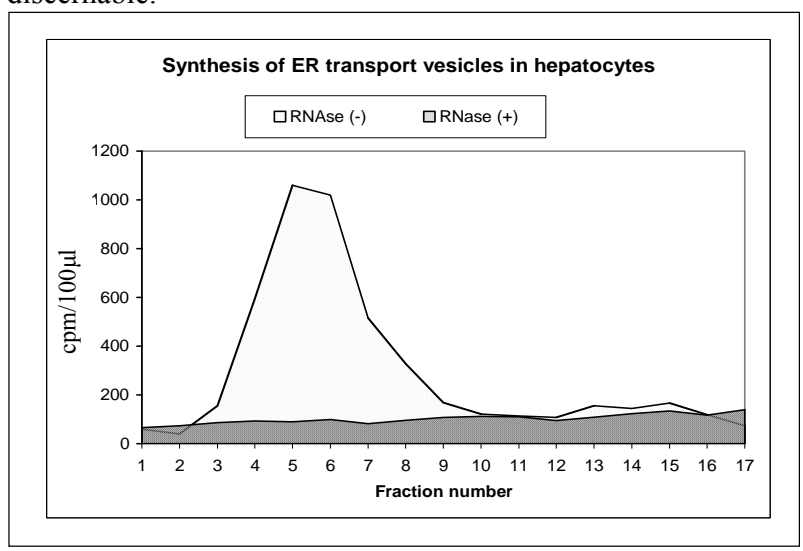

Figure 2. Synthesis of ER-transport vesicles is inhibited by treatment of the cytosol with RNase. The transport vesicles were generated by the procedure described in Methods. The vesicles that equilibrated between $45-50 \%$ sucrose (fraction 3-8) were recovered and their yield estimated by protein and radioactivity determination.

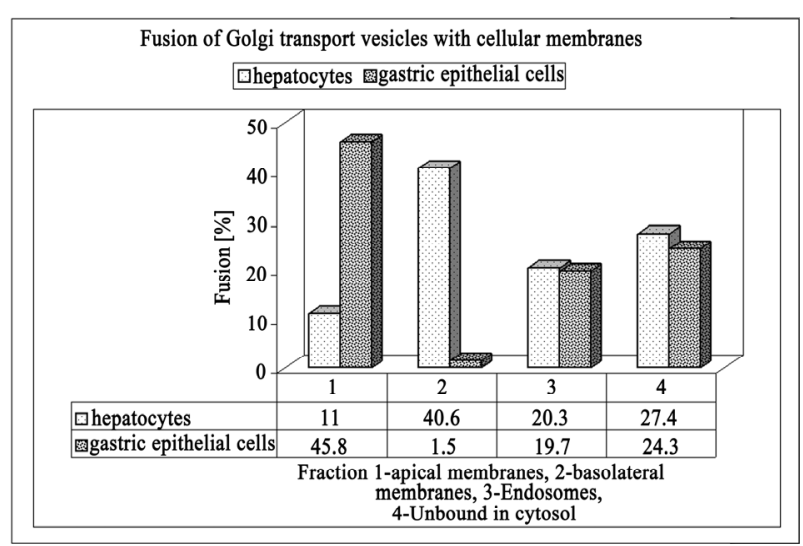

Figure 3. Fusion of Golgi transport vesicles with cell membranes is dictated by derivation of cytosol used for generation of ER transport vesicles. The experiments were initiated with ER-transport vesicles generated in the hepatocytes- or gastric mucosal epithelial cells cytosol and continued through generation of Golgi transport vesicles. The latter were subjected to fusion experiments with cold cell membranes, endosomes, ER, intact nuclei (IN) Outer Nuclear Membrane (ONM) and Inner Nuclear Membrane (INM). The experiments with fraction 4, the unbound Golgi vesicles recovered from cytosol were not fusing with ER, IN, ONM and INM are not presented.

association or fusion with ER, IN, ONM or INM. The possibility that the remaining vesicles are specific for fusion with mitochondria and lysosomes was not yet explored. The results of the described experiments demonstrate that RNA presence and derivation of the cytosol are crucial at ER stage and determine ER transport vesicles synthesis, composition and destination. Moreover, the assembled ER products consist of assortment of the vesicles that are destined to specific cell membrane sites and cell organelles, but do not return to ER or are involved in reconstitution of nuclear membranes.

The results on Golgi-localized fine adjustments in the ER-initiated biomembrane lipid composition of the gastric and hepatocytes derived vesicles reveal the principle differences in modifications of phospholipids that determine destination-specific biomembrane (Figure 4).

As shown in Figure 4(a), ER-vesicles'-delivered PI is subjected in Golgi to phosphorylation that affords three PIP derivatives. Thus, Golgi transport vesicles that fuse with gastric apical epithelial membrane contain PI and PI3P, and upon fusion LPIP is produced in the apical membranes (Figure 4(b)), the vesicles with affinity for endosomes contain PI and PI4P (Figure 4(c)). The remaining vesicles (Figure 4(d)) contain PI, PI4P, and yet unknown PIPs.

The Golgi transport vesicles generated from ER transport vesicles assembled in the hepatocytes-derived cytosol (Figure 5) contain PI and PIPs. The Golgi assembled mixture of vesicles displays largely PI4P and yet unidentified PIPs migrating on TLC between PI4P and

PI, while PI3P represented minor fraction (Figure 5(a)). Upon fusion, the basolateral membranes show presence of PI, PI4P and PIP2 (Figure 5(b)), whereas the apical membranes show presence of PI, PI3P and LPI3P (Figure 5(c)). The fraction of the vesicles which remains in the cytosol after fusion with cellular membranes contains mainly PI, PI4P and the fraction which co-migrates in the area occupied by PI (Figure 5(d)). Since in all experiments PI consisted of closely running doublet, it is possible that this separation of PIs is due to specific fatty acylation.

The labeling of ER transport vesicles with $\left[{ }^{3} \mathrm{H}\right]$ arachidonate and $\left[{ }^{3} \mathrm{H}\right]$ inositol allowed us to determine whether basolateral fusion of transport vesicles proceeds in similar fashion as apical and affords en bloc incorporation of the ER-assembled membrane. Results of these experiments are shown in Figure 6. The panel (a) shows TLC of the lipids extracted from basolateral membranes following fusion with arachidonate- and inositol-labeled Golgi transport vesicles. It demonstrates presence of labeled phospholipids, which in chromatographic separation utilizing solvent system consisting of ethyl acetate/isooctane/acetic acid/water (90/50/20/100, by vol.), appear as one fraction located at the origin of the plate (1), the presence of free arachidonate (2) and arachidonate-containing glycerides (3). The panel (b) presents separation of the phospholipids identified in panel (a) (1) and shows presence of LPIP (1), PC (2) PIPs $(3,4)$, PE (5), PA (6) and yet unknown phospholipid identified in position (7). Finally, panel (c) demonstrates spectrum of 
PIs that originates from $\left[{ }^{3} \mathrm{H}\right]$ inositol labeled PI of ER transport vesicles that reached basolateral destination. It
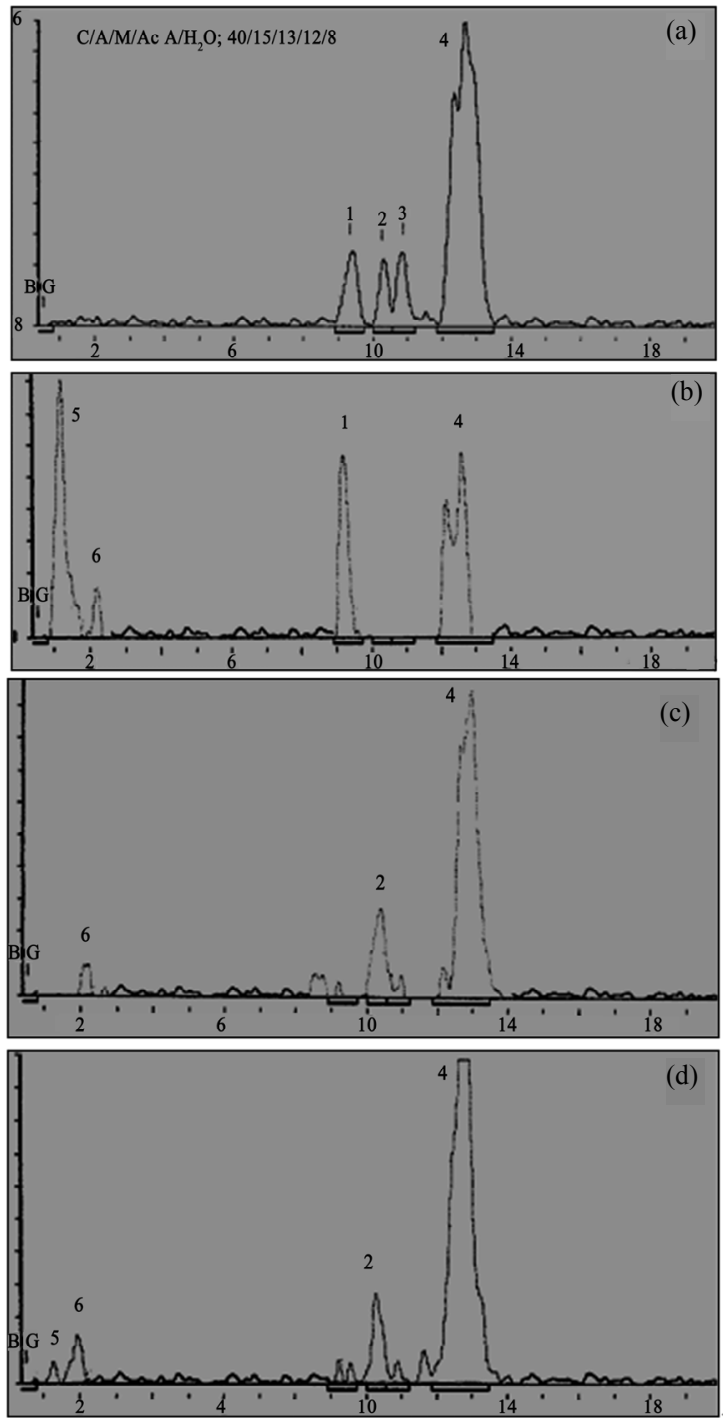

Figure 4. Phosphorylation of ER transport vesicles PI in Golgi. (a) Represents Golgi membranes after fusion with $\left[{ }^{3} \mathrm{H}\right]$ inositol labeled ER transport vesicles. Four PI-derived phosphatidylinositides were identified: 1-PI3P, 2-PI4P, 3-unknown PIP, and 4, -PI. (b) Golgi transport vesicles that fused with apical epithelial membrane contained 1-PI3P, 4-PI, 5-LPI3P and 6-PIP2. (c) Endosomal fraction following incubation with remaining Golgi transport vesicles contained 2-PI4P, 4-PI, and traces of PIP2. (d) Golgi transport vesicles that after incubation with endosomes remained free in cytosol consisted of 2-PI4P, 4-PI, and trace amount of 1,2- PIPs, 5-LPIPs and 6-PIP2. The separation and quantitative analyses of polyphosphoinositides depicted in this figure and Figure 5 and 6 was performed in one dimension on $1 \%$ ammonium oxalate impregnated $20 \times 20 \mathrm{~cm}$ thin layer chromatographic (TLC) plates developed in solvent system consisting of chloroform/acetone/methanol/ glacial acetic acid/water (40/15/13/12/8, by vol.) This procedure yielded quantitative separation of PI, PIPs, and LPIP, and PIP2. All TLC runs are compared with the elution profile of the $\left[{ }^{3} \mathrm{H}\right]$ inositol labeled polyphosphoinositides identified in Golgi following fusion with ER-inositol labeled transport vesicles depicted in (a).

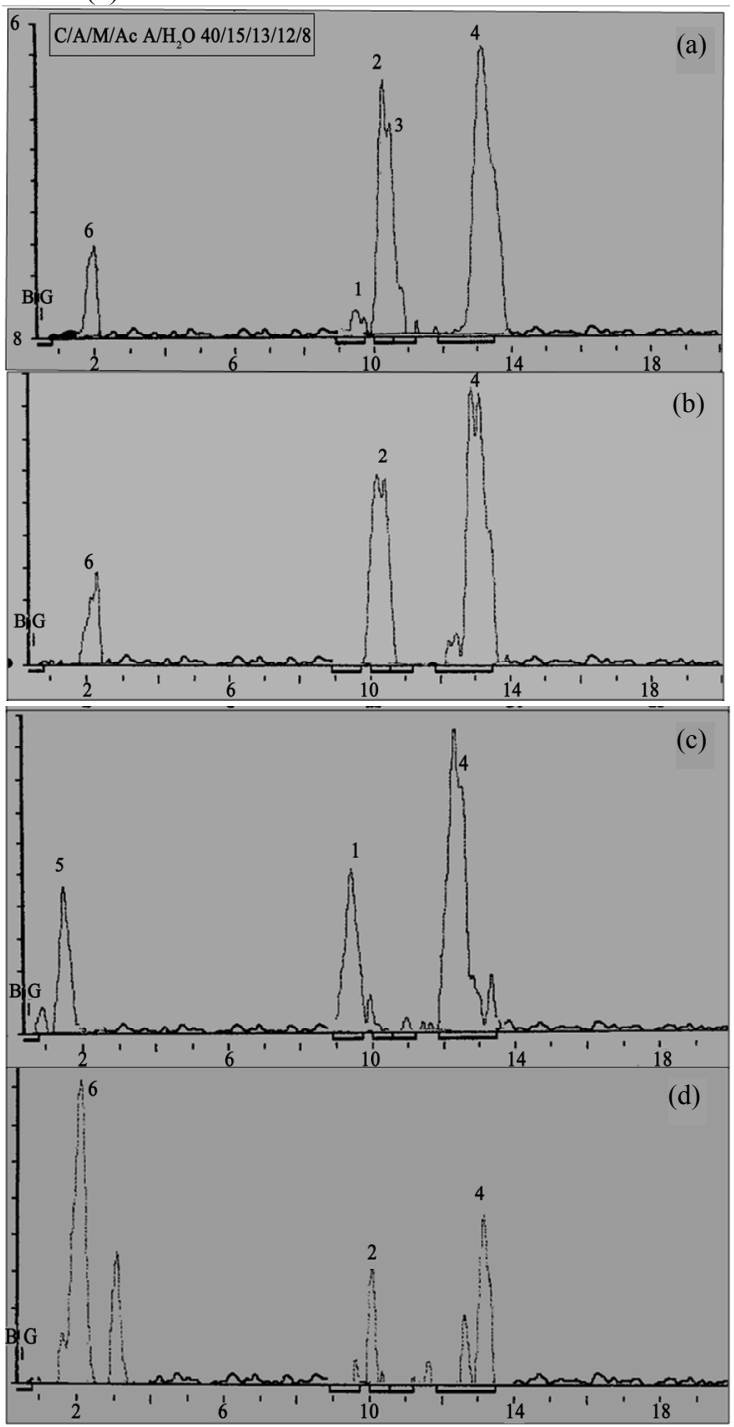

Figure 5. Phosphorylation of the hepatocytes ER transport vesicles PI in Golgi. (a) Represents phosphatidylinositides in Golgi-derived transport vesicles generated after fusion of $\left[{ }^{3} \mathrm{H}\right]$ inositol labeled ER transport vesicles with Golgi and consists of PIPs $(1,2,3)$, PI (4), and PIP2 (6). (b) Golgi vesicles that fused with basolateral membrane contained PI4P (2), PI (4) and PIP2 (6). (c) The apical membrane fraction showed presence of PI3P (1), PI (4) and LPI3P (5). (d) The unbound vesicles, which remained free in cytosol, contained traces of PI3P, PI4P (2), PIP2 (6) and unidentified inositol-labeled lipid migrating ahead of PIP2. The separation and identification of the labeled PI lipids was as described for Figure 4.

demonstrates presence of LPIPs (1), PIP2 (2,3), PIPs $(4,5)$, PI (6) and yet unknown inositol-labeled lipid identified in position (7). Thus, the results presented above provide evidence that basolateral fusion, just as apical, 
incorporates ER originated biomembrane targeted for basolateral membrane of the hepatocytes. It results in en bloc incorporation of the vesicular membrane (Figure 6)
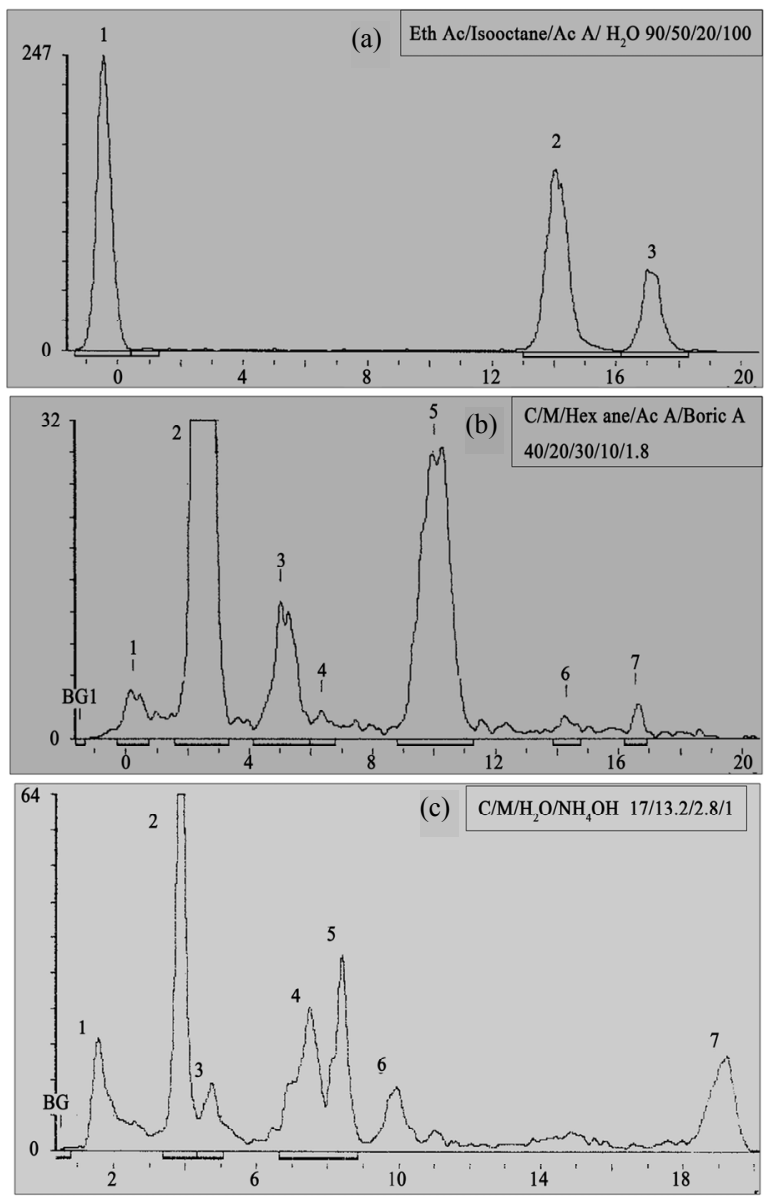

Figure 6. Identification of $\left[{ }^{3} \mathrm{H}\right]$ arachidonate- and/or $\left[{ }^{3} \mathrm{H}\right]$ inositol labeled lipids transferred via en bloc incorporated vesicular membrane initiated in ER to hepatocytes basolateral membrane. (a) Demonstrates fusion-induced release of arachidonate (2), the labeled phospholipids (1) and diacylglycerides (3). (b) Depicts TLC separation of the phospholipids shown in (A) position (1). The lipids consist of LPIP (1), PC (2), PIs (3), PS (4), PE (5), PA (6) and unknown (7). (c) The $\left[{ }^{3} \mathrm{H}\right]$ inositol-labeled lipids identified in basolateral membranes following en bloc fusion of ER-initiated transport vesicles consisted of LPIP (1), PIP2 (2), PIP2 (3), PIPs (4,5), PI (6) and unknown (7). The TLC solvent systems used are shown in each panel.

that is accompanied by generation of lysophospholipids ((1) in panel (b) and (c)) and arachidonate ((2) in panel (a)), incorporation of phospholipids identified in panel (b), and incorporation of the phosphorylation products of ER-synthesized PI demonstrated in panel (c).

While the cytosol derivation impacts synthesis, composition and final destination of ER transport vesicles, the Golgi-localized adjustments in spectra of transport vesicles lipid modification are also destination sensitive. In addition to PI phosphorylation, the synthesis of SM and GSL is destination-specific. So much so, that in the earlier studies we suggested that synthesis of the Cer determined the quantity and destination of the transport vesicles produced in gastric mucosal cells [15]. Indeed, present study support that notion since the amount of Cer in hepatocytes ER-transport vesicles is significantly smaller (Figure 1) and apparently is attributed to higher proportion of the vesicles with basolateral membrane destination (Figure 3).

After basolateral, apical and endosomal fusion, the significant amount of transport vesicles remaining in the cytosol led us to further experimentation on the specificity of transport in gastric mucosal epithelial cells and hepatocytes. In all experiments with Golgi transport vesicles derived from ER transport vesicles generated in the hepatocytes- or gastric cells-derived cytosol the remaining radiolabeled transport vesicles (Figure 3, fraction 4) in gastric cell cytosol or in hepatocytes cytosol would not fuse with ER or IN, ONM or INM (not shown). Since our earlier experiments with nuclear membranes provided evidence that these membranes biogenesis does not involve generation or release of transport vesicles, and that their lipid composition is different from Golgi transport vesicles, we concluded that the remaining in cytosol vesicles do not provide biomembrane for ER or nuclear membrane restitution. It is quite possible that the fraction consist of transporters dedicated for the mitochondrial or lysosomal membranes renewal and cargo acquisition. Based on the above described systematic fusion experiments with labeled ERand then Golgi-transport vesicles our strong contention is that restitution of the organellar membranes does not proceed through retrograde transport and re-utilization of the 'empty' vesicles. The process is unidirectional and that en bloc replacement of the ER-initiated membrane is the quintessential asset for the accurate reproduction of all cellular biomembranes.

Thus, from the studies presented here and the results published earlier we conclude that vesicular transport is dependent on the finely tuned synthesis of the cell-specific proteins and lipids that assemble in ER into the precise site-specific vesicular biomembranes [15-20]. Precise and explicit intercalation of the protein into the membrane is only plausible when the translation of mRNA and the synthesis of biomembrane proceed concomitantly and within the ER space capable to generate specific lipids and translate specific mRNAs. Therefore, the synthesis of ER transport vesicles is susceptible to RNase treatment and the protein and lipids of a new membrane determine the specific membrane maturation in Golgi and control directional transport from Golgi to specific sites of cell membrane and its organelles [25-27].

Together, these results allowed us to hypothesize that 
the substrates in the in situ medium (the cell cytosol) define the process of transport vesicles synthesis and delivery to the next organelle, since depletion of mRNA from either gastric or hepatocytes cytosol inhibits vesicles synthesis that fuse with Golgi, and the process is rescued with reinstatement of the initial cytosolic RNAs [17]. The synthesis of the intracellular biomembrane and the protein in ER must be initiated in a explicit site of the ER organelle, the site that is capable to receive nuclear transport of mRNAs to assemble appropriate quantity of secretory and constitutive proteins, specifically formulate biomembrane, and then deliver such packages en bloc to Golgi and to final destination points.

\section{DISCUSSION}

As recent reviews of the advances in understanding of the intracellular transport demonstrate, the investigations focus on the role of numerous proteins present on transport vesicles and their interaction with proteins projecting from the cellular membrane substrates [1-6,8,14,25-29]. The sorting mechanisms presume that for constitutive and regulated protein delivery individual proteins or their domains precisely control cellular compartmental organization and the exquisite and precise site-specific transfer of the products. In the illustrated concepts, the entire emphasis is placed on the proteins structure while the membrane lipids and the environment that they create for the protein function is not considered. It is assumed that the artificial lipid mixtures adequately substitute the native lipids because the protein binding through recognition of phospholipid-binding domain is observed and the transport of the protein to its designated site is achieved [3,10,28]. At the same time, the process allows to recover transporter and through retrograde movement use it for another round of ER products delivery [29]. These theory-driven assumptions provide illustrations of membranes with precisely intercalated proteins where minute changes in protein structure may impact process of fusion and membrane restitution, whereas the supporting experiments determine only the protein association with the membrane. Thus, it is arguable whether such pseudo membranes depict factual events and, whether mere closeness or even passive association of two artificial membranes is recreated with the fidelity that suffices the specific cargo delivery. It is questionable whether the fundamental principle that controls accuracy of the cell organelles and membranes restitution is controlled by protein alone [1,2,9,28,29]. The decisive factor that argues against the principle role of protein in cellular transport with the vesicles release and use for another round of transport is the vesicles synthetic complexity and the cellular need for metabolic compensation and repair of the membranes [30-32].
In our opinion, that is based on numerous studies of membrane biogenesis [15-20,24], the observed numerous vesicles that are assembled in ER and delivered to Golgi represent new cell-site specific transporters that are produced simultaneously with their protein cargo, and from Golgi are delivered to various sites for restitution and repair of the cell and its organelles [31,32]. Since, the $25 \%$ of transport vesicles that remain in cytosol after the major fractions fuse with apical, basolateral and endosomal membranes do not fuse with ER or nuclear membranes, they may represent transporters destined for mitochondria, lysosomes or other highly selective sites of the cell which in our experiments were not anticipated and presented [34,35]. The fact that radiolabeled lipids representing composition of these vesicles could not be identified/incorporated into hepatocytes or gastric cells derived ER or nuclear membranes argues against retrograde transport $[3,6,10,33]$. If the retrograde movement was viable process, the labeled lipids reflecting lipid composition of transport vesicles would not be evident in the membranes recovered after fusion, but instead be detectable in ER. In contrast, ER synthesized membrane lipids assembled into membrane of ER transport vesicles are carried to Golgi and from Golgi to various sites of the cell where they incorporate en bloc into membrane continuum.

As we have demonstrated earlier [15] and shown here following basolateral fusion, the cold membrane substrates contain exact mirror assemblies of the vesicles-derived radiolabeled lipids. The vesicles enriched in PI3P fuse with apical membrane, whereas the vesicles enriched in PI4P fuse with basolateral membrane, or display affinity for endosomes, or remain in cytosol and presumably constitute portion of the vesicles that display an affinity for other organelles. Moreover, the vesicular fusion with Golgi, apical and basolateral membrane (Figure 6) is accompanied with appearance of lysophospholipids and arachidonate, which suggests involvement of $\mathrm{PLA}_{2}$ in the membrane lysis and opening of transport vesicles $[15,18,36]$. The evidence that in Golgi the transport vesicles undergo progressive phospholipid maturation that undoubtedly is dictated by their destination and hence integral protein components, demonstrate how the cell membranes' specificity and transport is maintained. This in our opinion is the strongest argument against bidirectional transport and the vesicles as mere micro-containers for the transport.

As we found out through the experiments described here, the vesicles destination is decided in ER and dictated by the cell cytosol components. Therefore, ER transport vesicles produced in hepatocytes-derived cytosol do not fuse with gastric apical membrane, and conversely, the ER transport vesicles synthesized in gas- 
tric cell cytosol do not fuse with basolateral membrane of the hepatocytes. By concentrating on membrane lipid synthesis, the composition of the epithelial gastric and the hepatocytic membrane and by following the fusion of ER-initiated radiolabeled vesicles, we generated critical data that differentiate transport to the opposite domains of the epithelial cells and demonstrated impact of the cell cytosol on membrane composition and Golgispecific sorting.

Our study provides strong argument that Golgi sorting is not primary but the consequential event regarding the critical stages in development of transport vesicles and the maintenance of membrane compositional accuracy. By concentrating on the vesicular lipids we found out that specific membrane composition in terms of lipids and proteins are decisive in the process of vesicles fusion with apical or basolateral segment of cell membrane, and that synthesis of transport vesicle membrane is a ER-cotranslational, area-specific process. At the same time frame, the membrane integral proteins must be cotranslationally intercalated within actively synthesized lipids and together they reproduce fragment of the membrane that conforms to structural fidelity and determines transport vesicles destination.

Our previous studies, work of others [5-7,18,19,31] and the results presented here indicate that the restitution of ER and the cell nucleus membranes is not consequential of the vesicular pathway, and is not maintained through the retrograde vesicular transport. The lipids of ER and nucleus are not reflecting the composition of the apical cell membrane $[9,23,29,32,34,36]$. Also we could not substantiate a popular explanation of the subcellular membrane modification through Cer formation in plasma membrane [37], or transferring of the individual lipids from cytosol into ER [10,11], or nuclear membrane $[22,34]$. The incubation of ER, IN, ONM with cytosol enriched with sphingolipid extracts from Golgi vesicles, cell membrane rafts and caveolae, and apical cell membrane was not facilitating lipid integration into the membranes, and the lipids remained in cytosol [24]. Yet, our studies on nuclei and the inner and outer nuclear/ER membrane revealed feasible path associated with nuclear membrane restitution that was associated with transport of the cytosolic protein into the nucleus [24]. We have demonstrated that restitution of nuclear membranes is accomplished through the ability of endoplasmic reticulum to synthesize membrane lipids and utilization of the inositol phosphates (IPs) to generate PIPs and use them to transport cytosolic protein to nucleus. Appearance of labeled cytosolic protein and labeled PIPs in nucleus allowed us to speculate that the lipid synthesis-induced enlargement of ONM generates lateral movement of the nuclear membrane between nuclear pores and thus ac- complishes the transport of the cytosolic components into the nucleus and nuclear components to the cytosol. While we do not know whether nuclear membrane restitution proceeds through en bloc formation of the entirely new segments of the membrane, we are convinced that the restitution of the nuclear/ER and cellular and organellar membranes is accomplished through specific membrane lipid synthesis and lateral movement of newly ER-generated membrane.

In summary, our results allowed us to speculate that except ER/nuclear membrane, the principle, ER-initiated and cytosol-controlled vesicular membrane synthesis determines cellular transport and restitution. With this in mind we need to determine whether external signals releasing inositol phosphates (IPs) and provoking modification of the cell membrane stimulate nuclear processes that control transport of the sets of mRNA which will be translated into gamut of membranes that are specifically design to replace modified cell membrane, repair damage, or replace missing fragments used to generate endosomes, repair organelles affected by fission or deliver organelle specific enzymes. Are these events that are attributed to normal homeostatic repair and replacement of organelles and plasma membranes operational when singular protein translation is induced, inhibited or mutated [30,31,33-37]? Will singular gene deletion or genetic variation determine whether cell will lose its membrane characteristic features and be rejected from its environment as in cancer cells metastasis? If this were the case, would the incorporation of proper mRNA into cell cytosol restore normal cells characteristics? With this, an important task for future studies is to assimilate the concept that membrane restoration is achieved through en bloc incorporation of the precisely synthesized membrane segments in ER that predetermine apical, basolateral or organellar sorting. Without early ER coordination of the events, the complexity and diversity of the membrane renewal process is incomprehensible.

\section{REFERENCES}

[1] Sudhof, T.C. and Rothman, J.E. (2009) Membrane fusion: Grappling with SNARE and SM proteins. Science, 323, 474-477.

[2] Glick, B.S. and Nakano, A. (2009) Membrane traffic within the Golgi apparatus. Annual Review of Cell Development Biology, 25, 113-132.

[3] Lemmon, M.A. (2008) Membrane recognition by phospholipid binding proteins. National Review of the Molecular Cell Biology, 9, 99-111.

[4] Weisz, O.A. and Rodriguez-Boulan, E. (2009) Apical trafficking in epithelial cells: Signals, clusters and motors. Journal of Cell Science, 122, 4253-4266.

[5] Deborde, S., Perret, E., Gravotta, D., Deora, A., Salva- 
rezza, S., Schreiner, R. and Rodriguez-Boulan, E. (2008) Clathrin is a key regulator of basolateral polarity. Nature, 452,719-723.

[6] Folsch, H., Matilla, P.E. and Weisz, O.A. (2009) Taking the scenic route: Biosynthetic traffic to the plasma membrane in polarized epithelial cells. Traffic, 8, 972-981.

[7] Mellman, I. and Nelson, W.J. (2008) Coordinated protein sorting, targeting and distribution in polarized cells. $\mathrm{Na}$ tional Review of the Molecular Cell Biology, 9, 833-845.

[8] Walter, A.M., Wiederhold, K., Bruns, D., Fasshauer, D. and Sorensen, J.B. (2010) Synaptobrevin N-terminally bound to syntaxin-SNAP-25 defines the primed vesicle state in regulated exocytosis. Journal of Cell Biology, 188, 401-413.

[9] Chapmen, E.R. (2008) How does synaptotagmin trigger neurotransmitter release? Annual Review of Biochemistry, 77, 615-641.

[10] Idone, V., Tam, C. and Andrews, N.W. (2008) Two-way traffic on the road to plasma membrane repair. Trends in Cellular Biology, 18, 552-559.

[11] Van Meer, G., Voelker, D.R. and Feigenson, G.W. (2008) Membrane lipids: Where they are and how they behave. National Review of Cell Molecular Biology, 9, 112-124.

[12] Lingwood, D. and Simon, K. (2010) Lipid rafts as a membrane organizing principle. Science, 327, 46-50.

[13] Nohturfft, A. and Zhang, S.C. (2009) Coordination of lipid metabolism in membrane biogenesis. Annual Review of Cell Development Biology, 25, 539-566.

[14] Baron, W. and Hoekstra, D. (2010) On biogenesis of myelin membranes: Sorting, trafficking and cell polarity. Federation of European Biochemical Societies Letters, 584, 1760-1770.

[15] Slomiany, A., Nowak, P., Piotrowski, E. and Slomiany, B.L. (1998) Effect of ethanol on intracellular vesicular transport from Golgi to apical cell membrane: Role of phosphatidylinositol 3-kinase and phospholipase $A_{2}$ in Golgi vesicles association and fusion with the apical membrane. Alcoholism: Clinical and Experimental Research, 22,167-175.

[16] Slomiany, A. and Slomiany, B.L. (2003) Lipidomic processes in homeostatic and LPS- modified cell renewal cycle. Role of phosphatidylinositol 3-kinase pathway in biomembrane synthesis and restitution of apical epithelial membrane. Journal of Physiology and Pharmacology, 54, 533-551.

[17] Slomiany, A., Sano, S., Grabska, M., Yamaki, K. and Slomiany, B.L. (2004) Gastric mucosal cell homeostatic physiome. Critical role of ER-initiated membrane restitution in the fidelity of cell function renewal. Journal of Physiology and Pharmacology, 55, 837-860.

[18] Slomiany, A., Grzelinska, E., Yamaki, K., Palecz, D. and Slomiany, B.L. (1992) Biogenesis of endoplasmic reticulum transport vesicles transferring gastric apomucin from ER to Golgi. Experimental Cell Research, 201, 1669-1682.

[19] Slomiany, A., Grzelinska, E., Grabska, M., Yamaki, K., Tamura, S. and Slomiany, B.L. (1992) Intracellular processes associated with glycoprotein transport and processing. Archives of Biochemistry and Biophysics, 298, 167-175.

[20] A. Slomiany, A., Grabska, M., Piotrowski, E., Morita, M. and Slomiany, B.L. (1994) Intracellular processes asso- ciated with vesicular transport from endoplasmic reticulum to Golgi and exocytosis; Ethanol-induced changes in membrane biogenesis. Archives of Biochemistry and Biophysics, 310, 247-255.

[21] Slomiany, A., Grabska, M. and Slomiany, B.L. (2001) Essential components of antimicrobial epithelial barrier: Specific interaction of mucin with an integral apical membrane protein of gastric mucosa. Molecular Medicine, 7, 1-10.

[22] Slomiany, A.,Tamura, S., Grzelinska, E., Piotrowski, J. and Slomiany, B.L.(1992) Characterization of the "link" component of submandibular mucus glycoprotein. International Journal of Biochemistry, 24, 1003-1015.

[23] Brown, D.A. (2006) Lipid rafts, detergent-resistant membranes and raft targeting signals. Physiology, 21, 430-439.

[24] Slomiany, A., Grabska, M. and Slomiany, B.L. (2006) Homeostatic restitution of cell membranes. Nuclear membrane lipid biogenesis and transport of protein from cytosol to intranuclear spaces. International Journal of Biological Sciences, 2, 216-226.

[25] Fagone P. and Jackowski, S. (2009) Membrane phospholipid synthesis and endoplasmic reticulum function. Journal of Lipid Research, 50, S311-S316.

[26] Mayinger, P. (2008) Regulation of Golgi function via phosphoinositide lipids. Seminars in Cell and Developmental Biology, 20, 793-809.

[27] Prydz, K., Dick, G. and Tveit, H. (2008) How many ways through the Golgi maize? Traffic, 9, 299-304.

[28] Kawano, M., Kumagai, K., Nishijima, M. and Handa, K. (2006) Efficient trafficking of ceramide from endoplasmic reticulum to the Golgi apparatus requires VAMP-associated protein-interacting FFAT motif of CERT. Journal of Biological Chemistry, 281, 30279-30288.

[29] Van Meer, G., Halter, D., Sprong, H., Somerharju, P. and Egmond, M.R. (2006) ABC lipid transporters: extruders, flippases, or flopless activators? Federation of European Biochemical Societies Letters, 580, 1171-1177.

[30] Huizing, M., Helip-Wooley, A., Westbroek, W., Gunay-Aygun, M. and Gahl, W.A. (2008) Disorders of lysosome-related organelle biogenesis: Clinical and molecular genetics. Annual Review of Genomics and Human Genetics, 9, 359-386.

[31] Bryant, D.M. and Mostov, K.E. (2008) From cells to organs building polarized tissue. National Review of Molecular Cell Biology, 9, 887-901.

[32] Ladinsky, M.S., Mastronarde, D.N. and McIntosh, J.R (1999) Golgi structure in three dimensions: Functional insights from the normal kidney cell. Journal of Cell Biology, 144, pp. 1135-1149.

[33] Gill, D.J., Chia, J., Senewiratne, J. and Bard, F. (2010) Regulation of O-glycosylation through Golgi-to-ER relocation of initiation enzymes. Journal of Cell Biology, 189, 843-858.

[34] Tsai, N.P., Lin, Y.L. and Wei, L.N. (2010) Dual action of epidermal growth factor: extracellular signal-stimulated nuclear-cytoplasmic export and coordinated translation of selected messenger RNA. Journal of Cell Biology, 188, 325-338.

[35] Wymann, M.P. and Schneiter, R. (2008) Lipid signaling in disease. Nature Reviews. Molecular Cell Biology, 
9,162-176.

[36] Heringdorf, D.M. and Jacobs, K.H. (2007) Lysophospholipid receptors: Signalling, pharmacology and regulation of by lysophospholipid metabolism. Biochemica et Biophysica Acta, 1768, 923-940.
[37] Tam, C., Idone, V., Davlin, C., Fernandes, M.C., Flannary, A., He, X., Schuchman, E., Tabas, E. and Andrews, N.W. (2010) Exocytosis of acid sphingomyelinase by wounded cells promotes endocytosis and plasma membrane repair. Journal of Cell Biology, 189, 1027-1038. 